SLAC-PUB- 8046

December 1998

\title{
PHYSICS RESULTS FROM SLD USING THE CRID*
}

\author{
David Muller \\ Representing The SLD Collaboration** \\ Stanford Linear Accelerator Center \\ Stanford University, Stanford, CA 94309
}

\begin{abstract}
We review recent $Z^{0}$ physics results from SLD that use the Cherenkov Ring Imaging Detector for charged particle identification. The performance of the detector and likelihood method are described briefly. Several hadronization measurements are presented, including identified hadron production in events of different primary flavors, leading hadron production, and new correlation studies sensitive to details of both leading and nonleading hadron production. Identified $K^{ \pm}$have been used in conjunction with precision vertexing to study charmless and doubly charmed $B$-hadron decays. This combination has also been used to tag $b, \bar{b}, c$ and $\bar{c}$ jets, yielding precise measurements of $B^{0}-\bar{B}^{0}$ mixing and of the asymmetric couplings $A_{b}$ and $A_{c}$. Identified $K^{ \pm}$and $\Lambda^{0} / \bar{\Lambda}^{0}$ have been used to tag $s$ and $\bar{s}$ jets, yielding a measurement of $A_{s}$. The clean identified particle samples provided efficiently by the CRID allow the purities of these tags to be measured from the data, an essential ingredient for precision physics.
\end{abstract}

Presented at the $3^{\text {rd }}$ International Workshop on Ring Imaging Cherenkov Detectors, 15-20 November 1998, Ein-Gedi, Israel.

*Work supported in part by Department of Energy contract DE-AC03-76SF00515. 


\section{Introduction}

The SLD experiment [1] studies $Z^{0}$ bosons produced in $e^{+} e^{-}$annihilatons at the SLAC Linear Collider (SLC). A carrier of the electroweak interaction, the $Z^{0}$ boson decays into a fermion-antifermion $(f \bar{f})$ pair with probability predicted by the Standard Model (SM) electroweak couplings of the $Z^{0}$ to fermion $f$. The parity violation in this decay leads to an asymmetric distribution of the polar angle between the outgoing $f$ and the incoming $e^{-}$, which depends strongly on the $e^{+}$and $e^{-}$polarizations. The SLC electron beam is longitudinally polarized to a magnitude of $\sim 73 \%$ with a sign determined randomly for each beam pulse.

A key aspect of the SLD physics program is the measurement of total and asymmetric couplings, $R_{f}$ and $A_{f}$, for as many of the fundamental fermions $f$ as possible. Measuring $A_{f}$ requires both identifying $Z^{0} \rightarrow f \bar{f}$ events and determining the direction of the outgoing $f$ (as opposed to $\bar{f}$ ), which is challenging for the quarks, $f=u, d, s, c, b$, as they appear as jets of particles. $Z^{0} \rightarrow b \bar{b}$ and $c \bar{c}$ events can be identified by modern vertex detectors, using the $3(1) \mathrm{mm}$ average flight distance of the leading $B(D)$ hadron in each $b(c)$ jet. Quantities used to distinguish $b(c)$ from $\bar{b}(\bar{c})$ jets include the total charge of vertices or jets, and the charge of identified leptons or reconstructed $D$ mesons. The first two methods suffer from low analyzing power, and the other two from low efficiency. The charge of identified kaons is foreseen as a powerful method in future $B$ physics experiments, and we have recently pioneered its use for both $b$ and $c$ jets using our Cherenkov Ring Imaging Detector. The purity of a flavor tag can be measured from the data in $e^{+} e^{-}$annihilations using the anticorrelation between the $f$ and $\bar{f}$ in the event.

Light flavor $(u, d, s)$ jets are identifiable using their leading particles, and early work in this area is promising. We have used high-momentum strange particles to tag $s$ and $\bar{s}$ jets, measured the tag purities from the data, and made a measurement of $A_{s}$. We are

studying ways to saparate $u, \bar{u}, d$ and $\bar{d}$ jets. The tagging of light flavors would have a wide range of applications in high energy physics, from deep inelastic scattering, to jets from hadron-hadron collisions and studies of the decays of $W$-bosons, top quarks, Higgs bosons, and any new particle that is discovered.

In addition, the hadronic event samples at the $Z^{0}$ are of unprecendented size and purity, providing a unique opportunity to study the structure of hadronic jets and the decays of $B$ and $D$ hadrons in great detail. Jet formation is in the realm of non-perturbative QCD and is not understood quantitatively. The empirical understanding of jet structure is essential as jets are (will be) part of the signal for decays of $W^{ \pm}$bosons, $t$ quarks (any undiscovered heavy objects), as well as the background for these and other processes. Isolated jets have been identified with partons in order to make a number of tests of perturbative QCD. QCD tests, searches for new physics, and conventional physics studies would be more sensitive if we could distinguish jets of different origins (gluons, quarks, antiquarks). Jet structure in terms of inclusive properties of charged tracks has been studied extensively, however more theoretical and experimental input is needed, especially in the area of identified and reconstructed particles. In particular, the study of leading particles 
is needed for the development of light-flavor jet tags.

Decays of $B^{0}$ and $B^{+}$mesons have been studied extensively by experiments operating at the $\Upsilon(4 \mathrm{~S})$. The properties and mixture of $B$ hadrons produced at the $Z^{0}$ differ considerably from those at the $\Upsilon(4 \mathrm{~S})$, providing a number of opportunities for complementary studies of $B$ hadron decays [2], especially those involving identified particles for which the $\Upsilon(4 \mathrm{~S})$ experiments have limited momentum coverage.

To study this wide range of physics, the SLD includes a Cherenkov Ring Imaging Detector (CRID) designed to identify $\pi^{ \pm}, K^{ \pm}$and $\mathrm{p} / \overline{\mathrm{p}}$ over most of the momentum range, and leptons at low momentum, complemeting the electromagnetic calorimeter and muon detectors. The CRID design and performance are summarized in sec. 2. We then present a number of physics results in the areas of jet structure (sec. 3) and physics with flavor-tagged jets (sec. 4). Two unique studies [3] of $B$-hadron decays that benefit from SLD's excellent vertexing and particle identification are described separately in these proceedings [2].

\section{SLD CRID Performance}

The SLD CRID design and hardware performance are described in [4. Briefly, it is a large barrel detector covering the polar angle range $|\cos \theta|<0.68$, and comprising two radiator systems; liquid $\mathrm{C}_{6} \mathrm{~F}_{14}$ and gaseous $\mathrm{C}_{5} \mathrm{~F}_{12}+\mathrm{N}_{2}$ cover the lower and higher momentum regions, respectively. Cherenkov photons from the liquid (gaseous) radiator are focussed by proximity (spherical mirrors) onto one of 40 quartz-windowed time projection chambers (TPCs) containing ethane with $\sim 0.1 \%$ TMAE. Each single photoelectron drifts to a wire chamber where its conversion point is measured in three dimensions and used to reconstruct a Cherenkov angle $\theta_{c}$ with respect to each extrapolated track.

The average $\theta_{c}$ resolution for liquid (gas) photons was measured to be 16 (4.5) mrad, including errors on alignments and track extrapolation; the local resolution of 13 (3.8) mrad is consistent with the design value. The average number of detected photons per $\beta=1$ track was 16.1 (10.0) in $\mu$-pair events. In hadronic events, cuts to suppress spurious hits and cross-talk from saturating hits gave an average of 12.8 (9.2) accepted hits. The average reconstructed $\theta_{c}$ for $\beta=1$ tracks was 675 (58.6) mrad, independent of position

within the CRID and $\bar{\theta}_{c}^{\text {liq }}$ was constant in time. Time variations in $\bar{\theta}_{c}^{\text {gas }}$ of up to \pm 1.2 mrad were tracked with an online monitor and verified in the data.

Tracks were identified using a likelihood technique [5]. For each of the hypotheses $i=e, \mu, \pi, K, \mathrm{p}$, a likelihood $L_{i}$ was calculated based upon the number of detected photons and their measured $\theta_{c}$, the expected number and $\bar{\theta}_{c}$, and a background term. The background included overlapping Cherenkov radiation from other tracks in the event and a constant term normalized to the number of hits in the relevant TPC that were associated with no track.

Cuts on differences between the logarithms of these likelihoods, $\mathcal{L}_{i}=\ln L_{i}$, are optimized for each analysis. For example, in the analysis of charged hadrons (sec. 3) we 
considered only the hypotheses $i=\pi, K, \mathrm{p}$, and high purity was the primary consideration. We therefore applied a tight set of CRID quality cuts [6] (accepting $\sim 60 \%$ of the tracks with CRID information), and tracks with $p<2.5(p>2.5) \mathrm{GeV} /$ c were identified as species $j$ if $\mathcal{L}_{j}$ exceeded both of the other log-likelihoods by at least 5 (3) units. The matrix $\mathbf{E}$ of identification efficiencies is shown in fig. 11. The elements $E_{\pi j}$ and $E_{\mathrm{p} j}$ were determined from the data using tracks from selected $K_{s}^{0}, \tau$ and $\Lambda^{0}$ decays. The $E_{K j}$ were related to the measured elements using a detailed detector simulation. The bands in fig. 11 encompass the systematic errors on the efficiencies, determined from the statistics of the data test samples. The discontinuities correspond to Cherenkov thresholds in the gaseous radiator. The identification efficiencies peak near or above 0.9 and the pion coverage is continuous over $\sim 0.3-35 \mathrm{GeV} / \mathrm{c}$. There is a gap in the kaon-proton separation, $\sim 7-10$ $\mathrm{GeV} / \mathrm{c}$, and the proton coverage extends to the beam momentum. Misidentification rates are typically less than 0.03 , with peak values of up to 0.06 .

Many analyses required identifying $K^{ \pm}$with high efficiency and reasonable purity. A looser track selection was made ( $\sim 95 \%$ of CRID tracks) and a moderate cut, typically $\mathcal{L}_{K}-\mathcal{L}_{\pi}>3$, made against pions and leptons, along with a loose cut against protons, typically $\mathcal{L}_{K}-\mathcal{L}_{\mathrm{p}}>-1$. The efficiency for identifying true kaons is $\sim 70 \%$ for $1<p<30$ $\mathrm{GeV} / \mathrm{c}$; the pion (proton) misidentification rate depends strongly on momentum and can be as large as $12 \%(70 \%) . K^{ \pm}$samples of $70-90 \%$ purity are achieved. The power of this loose identification for reconstructing strange and charmed mesons is illustrated in figs. 2 and 3 .

The CRID has also been used in the identification of leptons. Alone, it provides efficient $e-\pi$ separation for $p<4 \mathrm{GeV} / \mathrm{c}$, and combined with the electromagnetic calorimeter gave improved $e^{ \pm}$identification for $p<8 \mathrm{GeV} /$ c. For $e^{ \pm}$from $B$ hadron decays, adding the CRID information resulted in a doubling of the efficiency of an optimized algorithm for a given purity. For $\mu^{ \pm}$, the CRID rejects $\pi^{ \pm}$for $2<p<4 \mathrm{GeV} / \mathrm{c}$, and $K^{ \pm}$, a substantial source of punchthrough, at all momenta. Reoptimizing the muon selection including CRID information increased the efficiency by $\sim 10 \%$ at all $p$, and the purity by $\sim 40 \%$ for $2<p<4 \mathrm{GeV} / \mathrm{c}$ and $5-30 \%$ at higher momenta. 


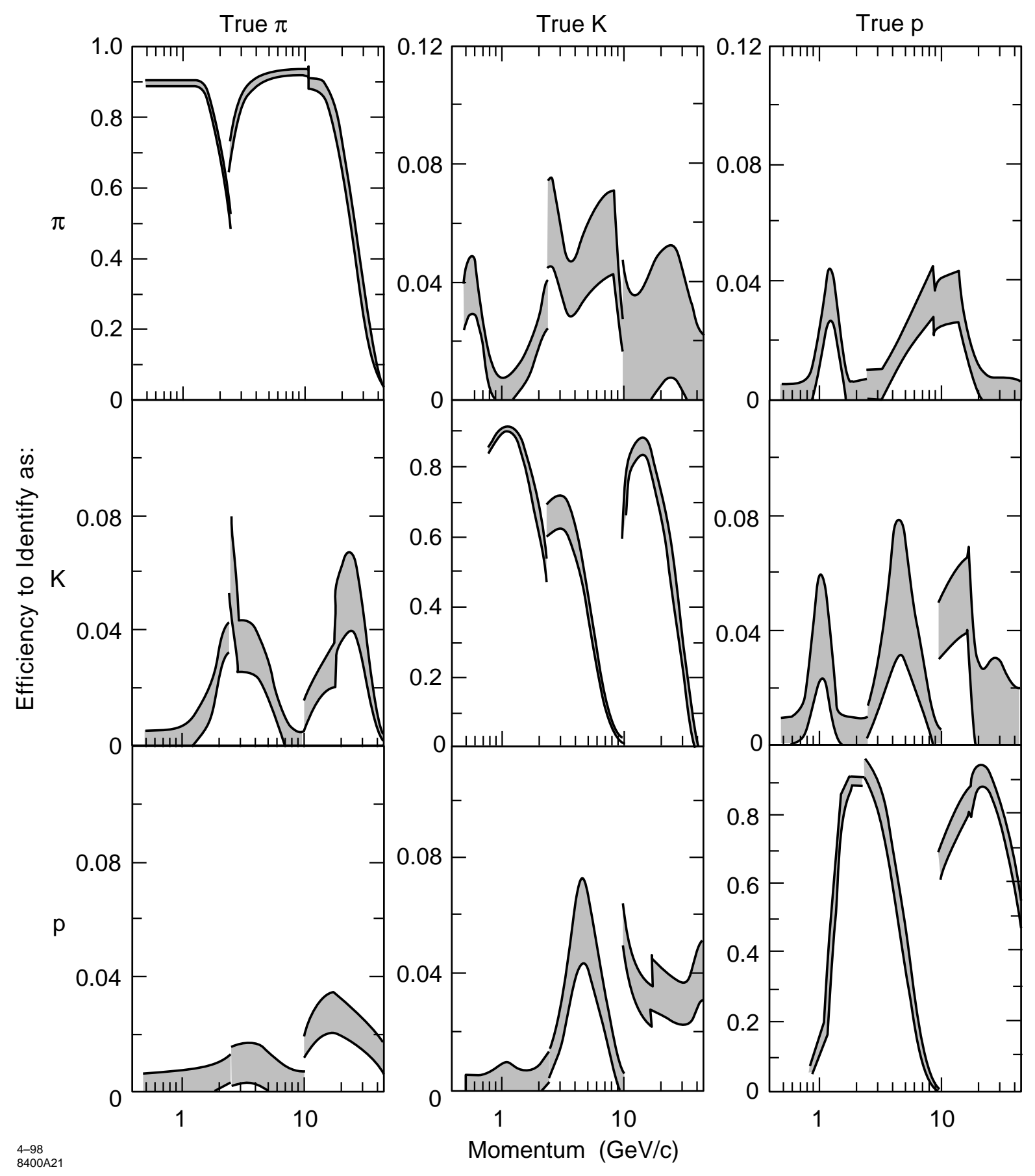

Figure 1: Calibrated identification efficiencies for tracks used in the charged hadron analysis. 

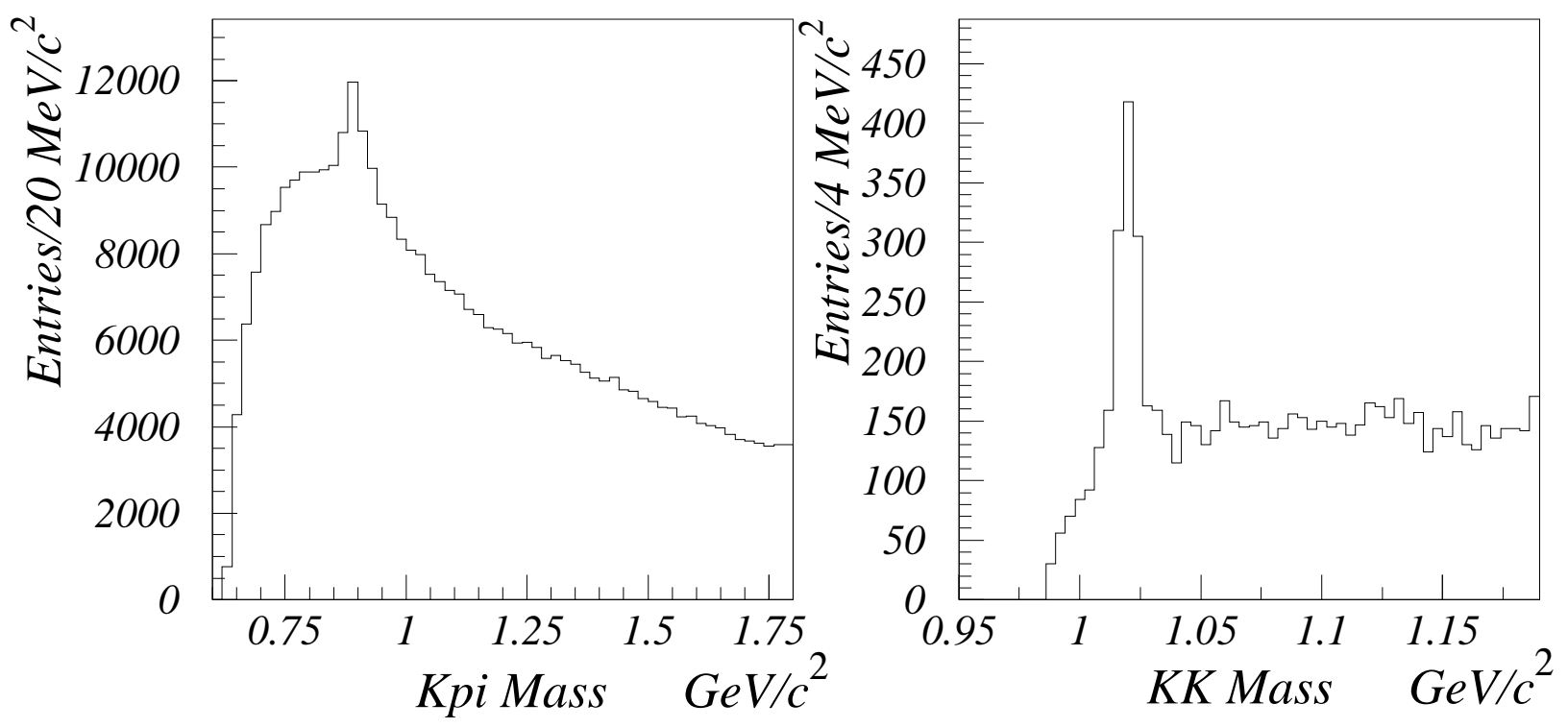

Figure 2: Distributions of invariant mass using loosely identified kaons (see text) showing signals for the $K^{* 0}(890)$ and $\phi(1020)$.
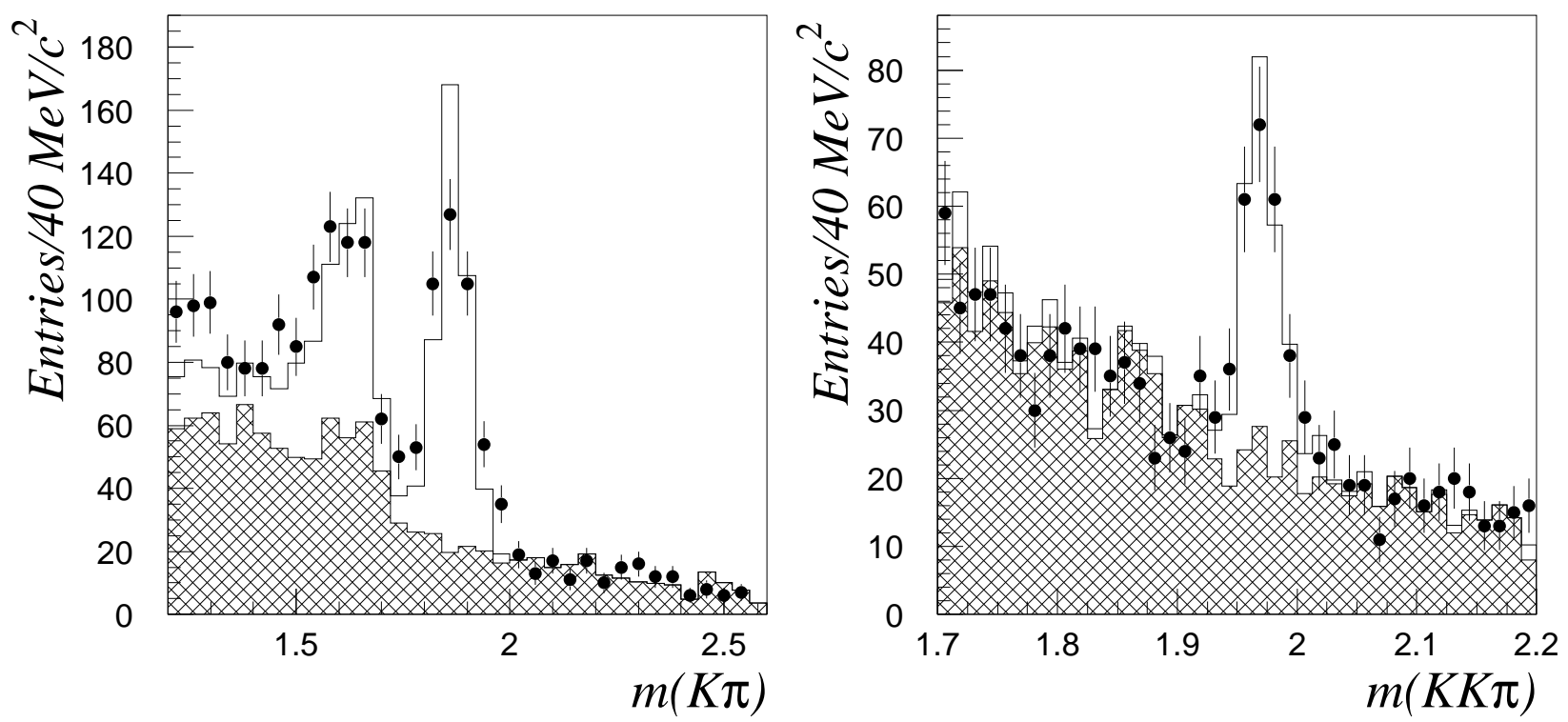

Figure 3: Invariant mass distributions for tracks forming secondary vertices (dots) showing signals for the $D^{0}$, including the satellite peak, and $D_{s}$ mesons. The simulated (background) distributions are shown as (hatched) histograms. 


\section{Hadronization Physics}

Inclusive properties of the charged tracks and photons in jets have been studied extensively in $e^{+} e^{-}$annihilations. Studies of specific identified particles at lower energies had low statistics and incomplete momentum coverage, but were able to observe the production of baryons, vector mesons and strange mesons and baryons, and to study mechanisms for strangeness and baryon number conservation through correlations. The large samples at the $Z^{0}$ have allowed much more detailed studies [7], including the recent observations of tensor mesons and orbitally excited baryons.

We have studied the production of seven identified particle species in hadronic $Z^{0}$ decays [6]. Charged $\pi^{ \pm}, K^{ \pm}$and $\mathrm{p} / \overline{\mathrm{p}}$ identified as described in sec. 2 were counted as a function of momentum and these counts unfolded using the inverse of the identification efficiency matrix (fig. 1) to yield production cross sections as a function of momentum. The neutral strange vector mesons $K^{* 0}$ and $\phi$ were reconstructed in their $K^{+} \pi^{-}$and $K^{+} K^{-}$modes, respectively, using the loose kaon selection (sec. 2). Production cross sections were extracted from fits to the invariant mass distributions (see fig. 2). These cross sections, along with similar measurements for $K^{0}$ and $\Lambda^{0} / \bar{\Lambda}^{0}$ [6], are shown in fig. 4 .

These measurements cover a wide momentum range with good precision. Similar inclusive measurements have been made [0] at the $Z^{0}$ by DELPHI using RICH particle identification, and by ALEPH and OPAL using $\mathrm{d} E / \mathrm{d} x$. The measurements are consistent, have comparable precision and, between the two methods, cover the entire momentum range. The clean samples available from the CRID compensate for the much higher statistics at LEP, and also allow smaller systematic errors in some cases, most notably

the $K^{* 0}$, for which the background is high and contains large contributions from reflections of resonances decaying into $\pi^{+} \pi^{-}$.

The CRID efficiency and purity are especially useful when the data are divided into smaller samples and additional levels of unfolding are required. We divided the hadronic events into $b$-, $c$ - and light-flavor $(u, d, s)$ samples [6], repeated the above analyses on each, and unfolded the results to yield production cross sections in these three flavor categories. Substantial differences are observed, as expected from the known production and decay properties of the leading heavy hadrons. The results for the light-flavor sample are shown in fig. 5, where coverage and precision comparable to that of the flavor-inclusive sample are evident. This measurement provides a more pure way of looking at hadronization at a fundamental level. We have found these results to be consistent with the limited predictions of perturbative QCD [6], and compared them with the predictions of three hadronization models (see fig. 5). All describe the data qualitatively; differences in detail include: all models are high for low-momentum kaons; the JETSET model is high for the vector mesons and protons at all momenta; the HERWIG model and, to a lesser extent, the UCLA model show excess structure at high momentum for all particle species; no model is able to reproduce the $\sim 10 \%$ difference between $K^{ \pm}$and $K^{0}$ production. These discrepancies have been observed previously [7], however our measurement demonstrates unambiguously that they are in the hadronization part of the model and not in the simula- 


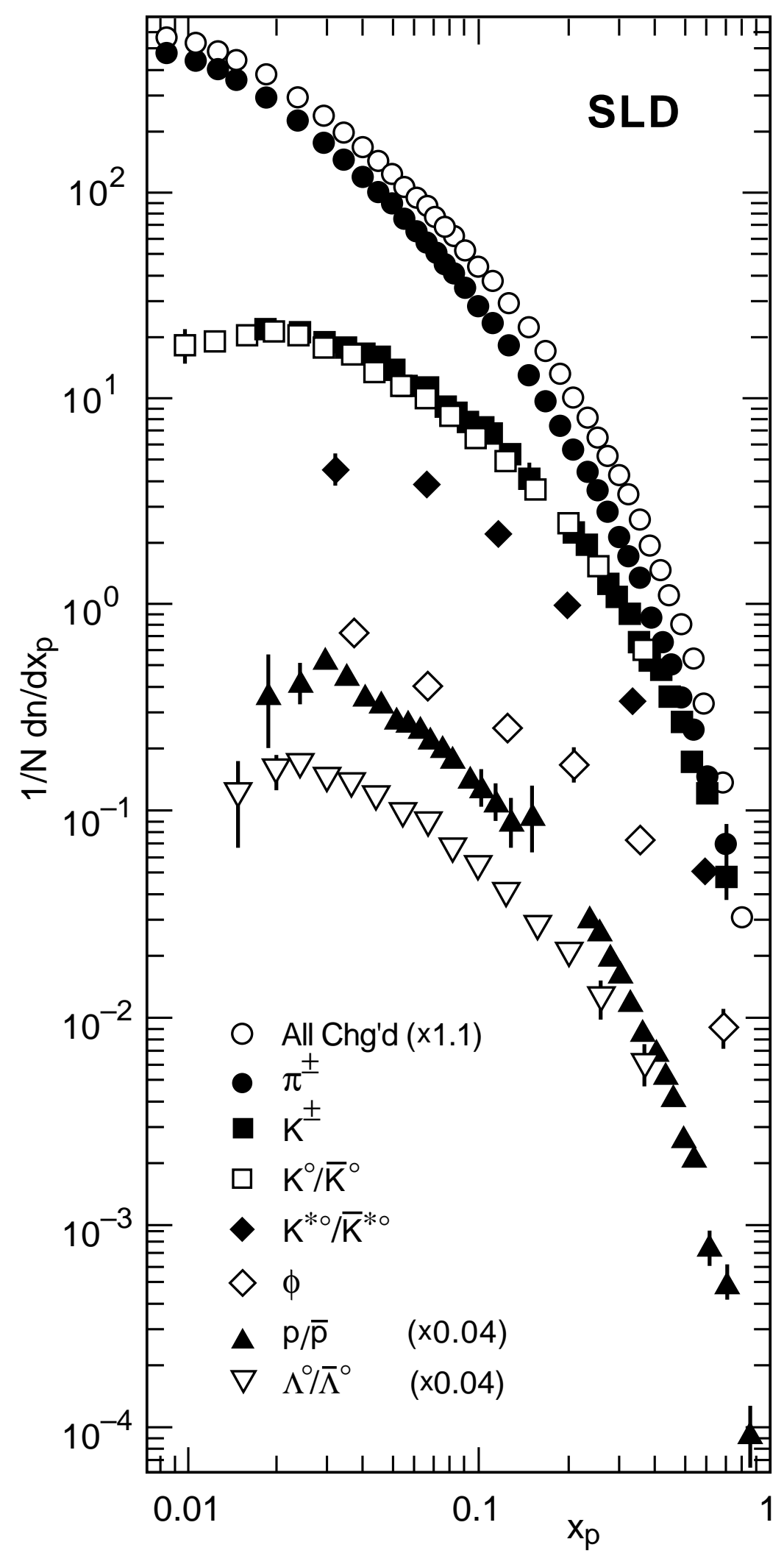

Figure 4: Differential production cross sections per hadronic $Z^{0}$ decay per unit $x_{p}$ for all charged particles and seven identified hadron species. 


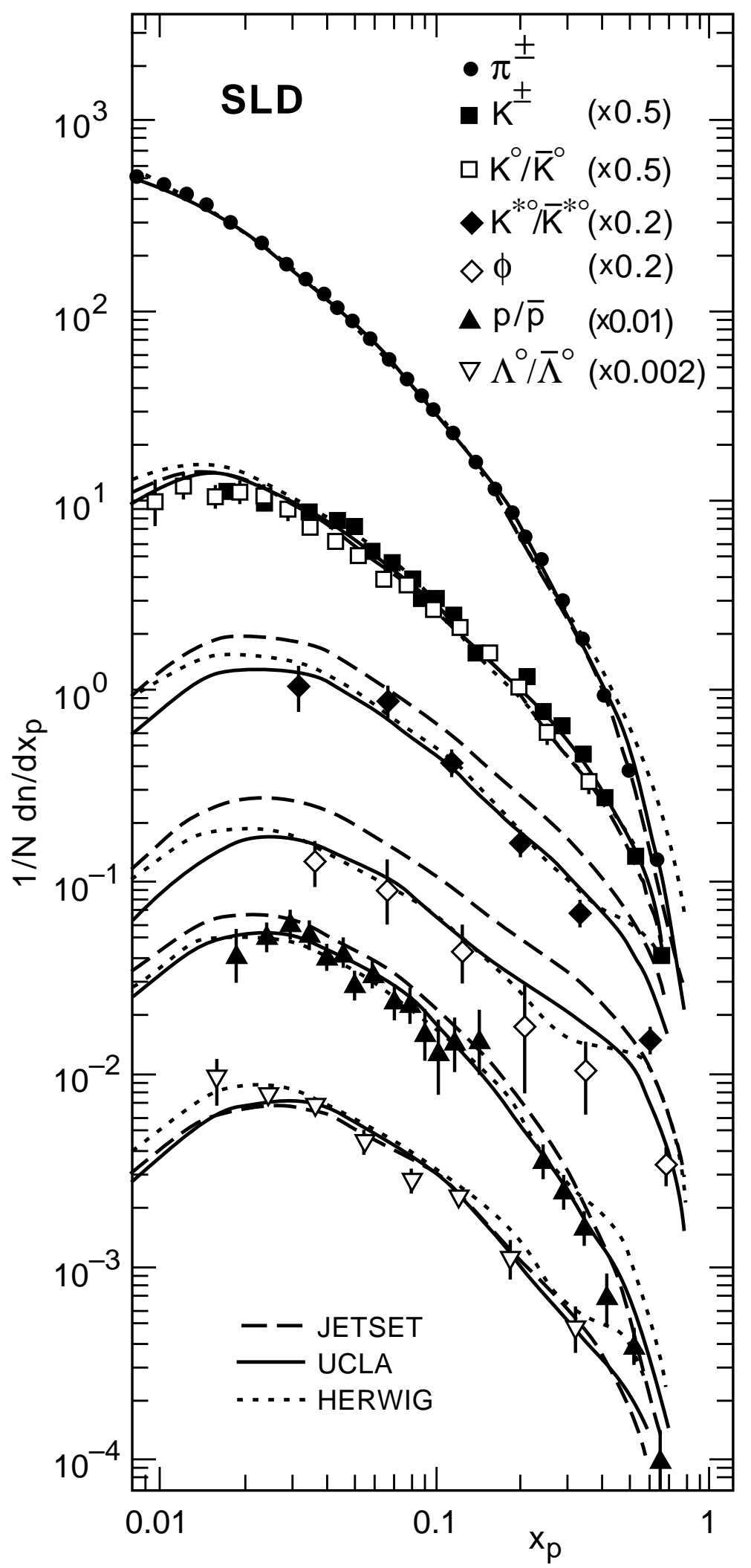

Figure 5: Differential production cross sections per light-flavor hadronic $Z^{0}$ decay, compared with the predictions of three hadronization models. 
tion of heavy hadron production and decay. We have found additional minor discrepancies in the heavy flavor events [6].

The events in the light flavor sample can be divided further into quark hemispheres and antiquark hemispheres using the electron beam polarization, providing a unique study of leading particle effects [8, []]. For events with thrust axis $|\cos \theta|>0.2$, the forward (backward) thrust hemisphere is tagged as the quark jet if the beam is left-(right-)polarized, and the opposite hemisphere tagged as the antiquark jet. The SM predicts a quark purity of $73 \%$. The set of hadrons in quark jets plus their respective antihadrons in antiquark jets were analyzed to yield cross sections $\sigma_{h}$ for hadrons in light quark jets. Similarly, the remaining hadrons yielded antihadron cross sections $\sigma_{\bar{h}}$. The corrected normalized production differences $D_{h}=\left(\sigma_{h}-\sigma_{\bar{h}}\right) /\left(\sigma_{h}+\sigma_{\bar{h}}\right)$ are shown in fig. 6.

At low momentum, hadron and antihadron production are consistent. At higher momentum there is an excess of baryons over antibaryons, as expected from leading baryon production (a baryon contains valence quarks, not antiquarks). The large excess of pseudoscalar and vector antikaons over kaons at high momentum is evidence both for leading kaon production, and for the dominance of $s \bar{s}$ events in producing leading kaons. No significant leading particle signature is visible for pions.

CRID type particle identification greatly enhances studies of pairs of hadrons in the same event. We have analyzed [9] correlations in rapidity $y=0.5 \ln \left(\left(E+p_{\|}\right) /\left(E-p_{\|}\right)\right)$, where $E\left(p_{\|}\right)$is the energy (momentum projection onto the thrust axis) of the hadron, between pairs of identified $\pi^{ \pm}, K^{ \pm}$and $\mathrm{p} / \overline{\mathrm{p}}$ in light-flavor events. We compared the distribution of $\Delta y=\left|y_{1}-y_{2}\right|$ for identified $K^{+} K^{-}$pairs with that for $K^{+} K^{+}$and $K^{-} K^{-}$ pairs. The latter are expected to be uncorrelated, and the difference between the two distributions illuminates strangeness production in the hadronization process. We observe 99] a large difference at low values of $\Delta y$; this 'short range' correlation indicates that the conservation of strangeness is 'local', that is, a strange and an antistrange particle are produced close to each other in the phase space of the jet. Similar effects for $\mathrm{p} \overline{\mathrm{p}}$ and $\pi^{+} \pi^{-}$pairs indicate local conservation of baryon number and isospin, respectively. Such effects have been observed previously, however the CRID has allowed the study of the shape and range of the correlations in detail and at many momenta, in particular we have verified the scale-invariance of the range. We have also observed short-range correlations between opposite-charge $\pi K, \pi \mathrm{p}$ and $K$ p pairs; they are relatively weak, and high purity is required to separate them from the large $\pi \pi$ background. They suggest charge-ordering of all particle types along the $q \bar{q}$ axis and provide new tests of fragmentation models.

We also expect correlations at long range due to leading particles, e.g. an $s \bar{s}$ event may have a leading $K^{-}$in the $s$ jet and a leading $K^{+}$in the $\bar{s}$ jet that have a large $\Delta y$. We have studied pairs of identified hadrons that both have $p>9 \mathrm{GeV} / \mathrm{c}$. Their $\Delta y$ distributions are shown in fig. 7; the $p$ cut separates each distribution into two parts, one $(\Delta y<2)$ comprising pairs in the same jet and the other $(\Delta y>3)$ comprising pairs in opposite jets of the event. Strong $K^{+} K^{-}$correlations are seen at both short and long range, as expected. For baryon and pion pairs, any long-range correlation will be diluted by the short-range correlation - a high-momentum leading baryon will always be 


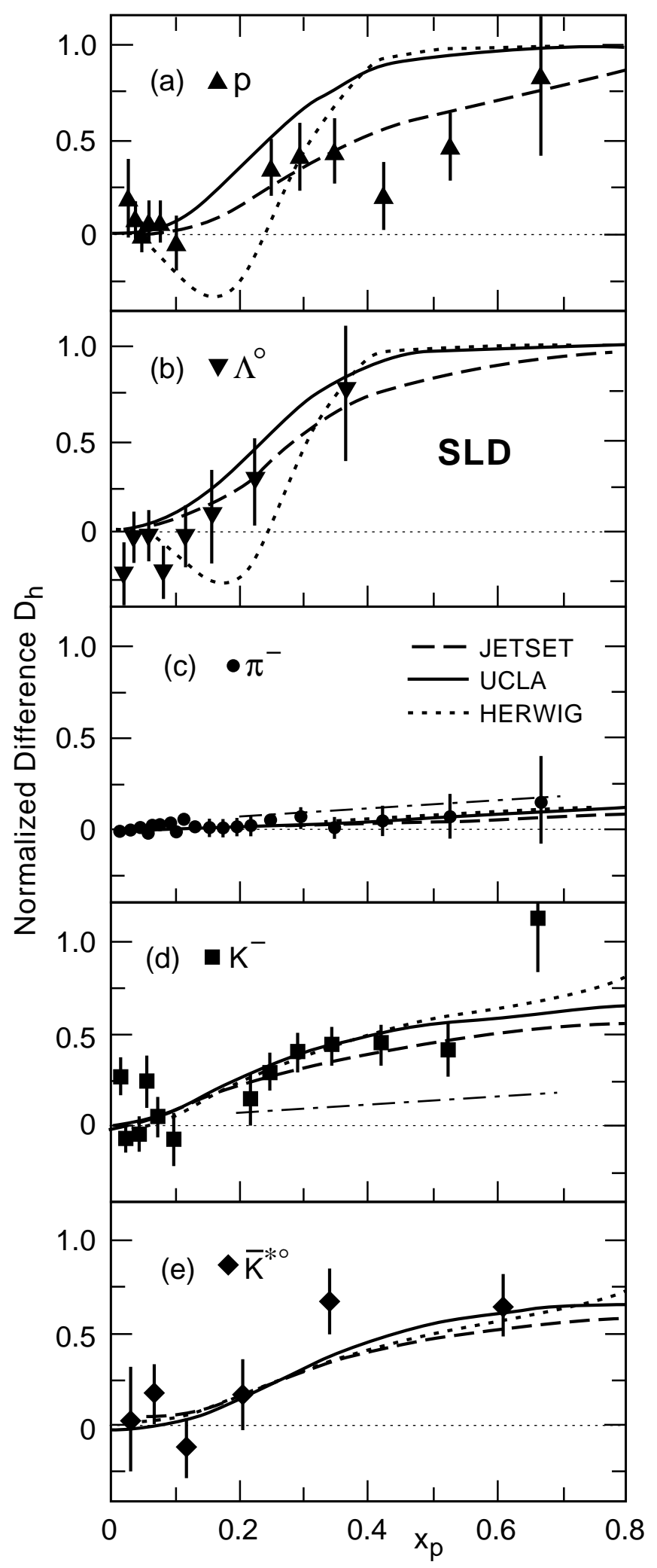

Figure 6: Normalized differences between hadron and antihadron production in uds jets; the predictions of the three fragmentation models. 


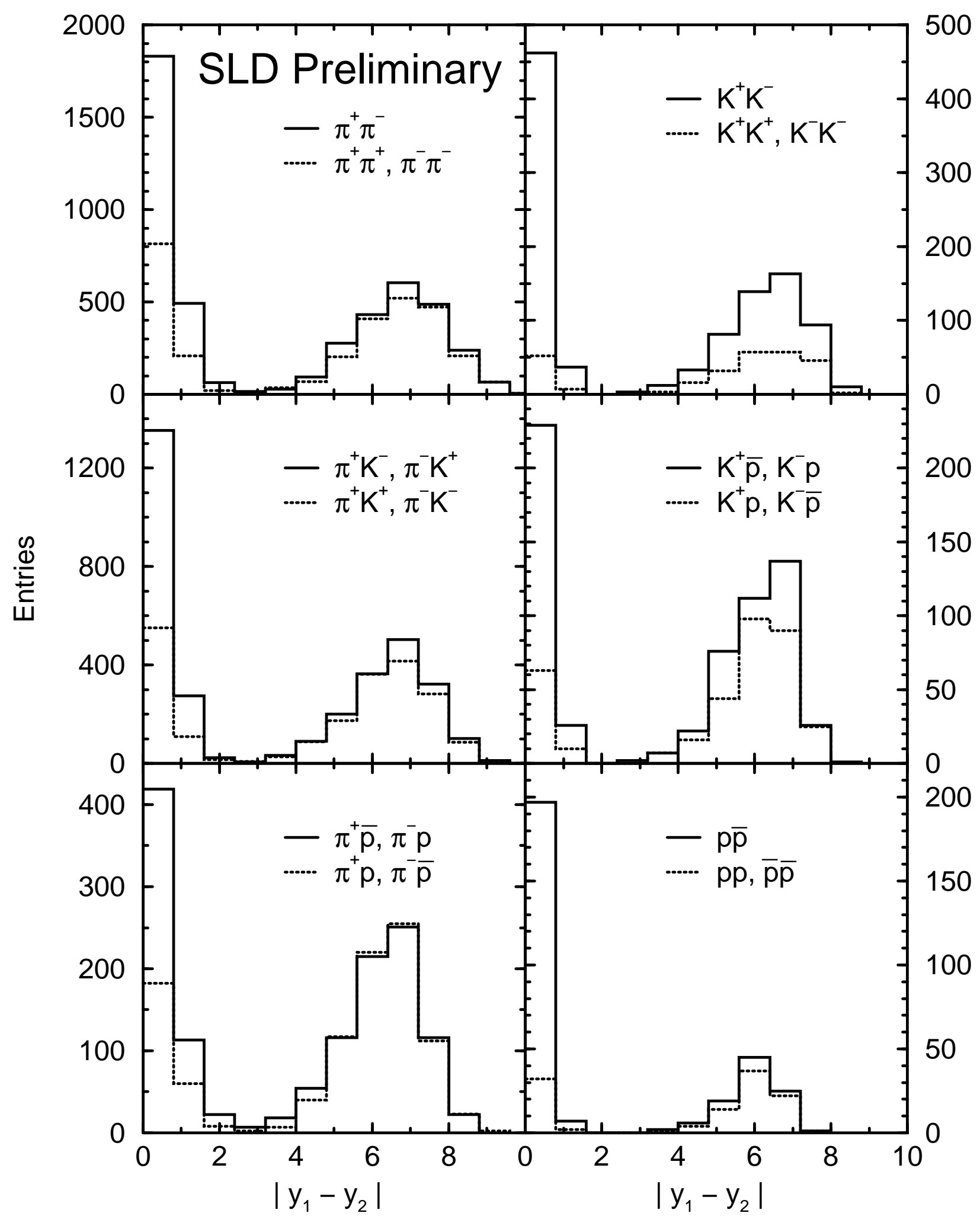

Figure 7: $\quad|\Delta y|$ distributions for opposite- (histograms) and same-charge (dashed histograms) pairs in which both tracks have $p>9 \mathrm{GeV} / \mathrm{c}$. 
accompanied by a subleading antibaryon, also with high momentum. We do not observe a long-range correlation for $\mathrm{p} \overline{\mathrm{p}}$ pairs, however we do observe a significant correlation for $\pi^{+} \pi^{-}$pairs, providing direct evidence for leading pion production. There are also significant correlations for $\pi K$ and $K$ p pairs, but not for $\pi$ p pairs. These cross terms provide new information on leading particle production in jets of different flavors, which will eventually allow the use of high momentum identified particles to separate $u \bar{u}, d \bar{d}$ and $s \bar{s}$ events from each other.

Using the beam polarization to select the quark hemisphere in each event, we have performed a new study [9] of rapidities signed such that $y>0(y<0)$ corresponds to the (anti)quark direction. A pair of identified hadrons can then be ordered, for example by charge to form the ordered rapidity difference $\Delta y^{+-}=y_{+}-y_{-}$. A positive value of $\Delta y^{+-}$ indicates that the positively charged hadron is more in the direction of the primary quark than the negatively charged hadron. The distribution of $\Delta y^{+-}$can be studied in terms of the difference between its positive and negative sides. We observe a large difference for $K^{+} K^{-}$pairs at long range due to leading kaon production in $s \bar{s}$ events. A significant difference at short range for $\mathrm{p} \overline{\mathrm{p}}$ pairs at all $p$ is direct evidence that the proton in a correlated $\mathrm{p} \overline{\mathrm{p}}$ pair prefers the quark direction over the antiquark direction.

\section{Quark Flavor Tagging and Electroweak Physics}

The identification of the flavor of the quark that initiated a hadronic jet is required for a wide variety of physics. The development of precision vertex detectors has enabled the pure and efficient tagging of $b / \bar{b}$ and $c / \bar{c}$ jets, leading to a number of precise measurements in fixed-target and $e^{+} e^{-}$annihilation experiments and the understanding of top quark production at hadron colliders.

For many measurements it is also necessary to distinguish $b$ from $\bar{b}$ or $c$ from $\bar{c}$ jets. The use of charged kaons is envisioned for this purpose in several future $B$ physics experiments, and has recently been pioneered by SLD. We present examples of its use for both $b$ and $c$ physics, which rely on the CRID for high efficiency and purity that is measurable from the data.

The identification of light flavor jets is a field in its infancy. The three light flavors can be separated from $b / \bar{b}$ and $c / \bar{c}$ jets by the absence of a secondary vertex in the jet; the only known way to distinguish them from each other is by identifying the leading particle in the jet cleanly. Little experimental information on leading particle production in light flavor jets exists (much of which appears in sec. 3 above), making the measurement of tagging purities and analyzing powers from the data essential. We present a measurement of $A_{s}$ and discuss prospects for light-flavor tagging in general. 


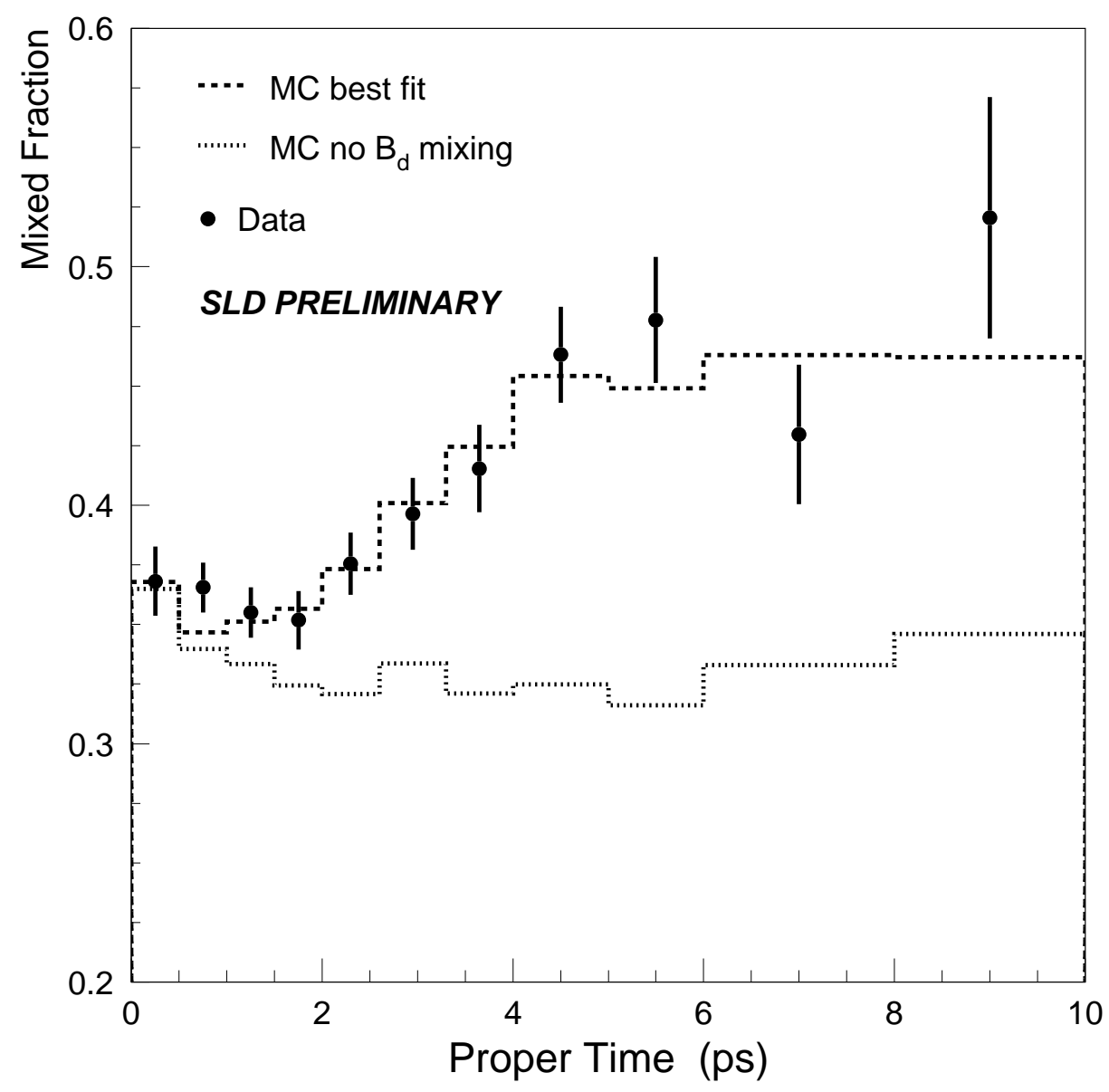

Figure 8: Mixed fraction as a function of proper decay time for tagged $B$ decay vertices.

\section{A. $B^{0}-\bar{B}^{0}$ Mixing}

To measure the time dependence of $B^{0}-\bar{B}^{0}$ mixing, one must tag neutral $B$ hadrons and determine their flavor $(B$ or $\bar{B})$ at both production and decay time. We first selected a sample of high-mass secondary vertices [10] (98\% B-purity, $\sim 40 \% B_{d}^{0}$ ) and reconstructed the proper decay time $\tau$ of each. The flavor at production was determined by a combination of the beam polarization and the charges of the tracks, identified $K^{ \pm}, e^{ \pm}$and $\mu^{ \pm}$ in the opposite hemisphere, with a correct-sign fraction of $88 \%$. The flavor at decay time was determined from the charge of any identified $K^{ \pm}$attached to the secondary vertex, with a correct-sign probability $P_{\text {corr }}=77 \%$.

The fraction of events classified as mixed (different flavors at production and decay) is shown as a function of $\tau$ in fig. 8. A clear increase with time is evident, which is the signal for $B_{d}^{0}-\bar{B}_{d}^{0}$ mixing. A fit to the data yielded a $6 \%$ measurement of the mass difference $\Delta m_{d}$. There are several other measurements of $\Delta m_{d}$ on the market with similar precision, including three from SLD, however this is the only one using the $K^{ \pm}$ tag, providing valuable complementarity.

There is considerable interest in $B_{s}^{0}-\bar{B}_{s}^{0}$ mixing, for which there are currently only 


\begin{tabular}{|c|c|c|c|c|c|c|c|}
\hline $\begin{array}{l}\text { Flavor Tag } \\
\text { Method }\end{array}$ & $\begin{array}{l}\text { Rel. } \\
\text { Eff. }\end{array}$ & $\begin{array}{l}\text { Correct Sign } \\
\text { Fraction b/c }\end{array}$ & $\begin{array}{c}\text { Corr. Frac. } \\
\text { Measmt. }\end{array}$ & $\begin{array}{l}\text { Error } \\
\text { Now }\end{array}$ & Error on $A_{b}$ & \multicolumn{2}{|c|}{ Error on $A_{c}$} \\
\hline$Q$ & 1.0 & $\sim 0.6,|Q|$ & & & & 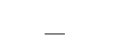 & - \\
\hline Id'd $K^{ \pm}$ & 0.4 & $\sim 0.75 / 0.90$ & cle & 0 . & 0.040 & 0.050 & 0.036 \\
\hline lepton & 0.1 & $\sim 0.90 / 0.95$ & stat. ltd. & 0.069 & 0.044 & 0.110 & 0.070 \\
\hline $\operatorname{Rec}{ }^{\prime} d D^{(*)}$ & 0.01 & $\sim 0.85 / 1.00$ & stat. ltd. & 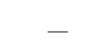 & 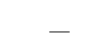 & 0.072 & 0.044 \\
\hline
\end{tabular}

Table 1: Summary of SLD heavy flavor asymmetry measurements

lower limits on the frequency. Our $K^{ \pm}$charge tag is insensitive to the $B_{s}$ flavor, since the $B_{s}^{0}$ decay contains both a $K$ and $\bar{K}$ meson, which is a feature of the above $B_{d}^{0}-\bar{B}_{d}^{0}$ mixing measurement. However, the measurement of $B_{s}^{0}-\bar{B}_{s}^{0}$ mixing requires a strongly enriched $B_{s}$ sample, for which the CRID is quite useful (see fig. 3). Also, the subleading $K^{-}$produced in association with a $\bar{B}_{s}^{0}$ meson can be used as an initial state tag, as demonstrated in a $B_{s}$ production measurement by DELPHI [目].

\section{B. Heavy Flavor Asymmetries}

In order to measure the asymmetric coupling $A_{q}$ of the $Z^{0}$ to quarks of flavor $q$ it is necessary to identify the event flavor as $Z^{0} \rightarrow q \bar{q}$ and to determine the polar angle $\theta_{q}$ of the primary $q$. The event thrust axis provides a good estimate of $\left|\cos \theta_{q}\right|$, however it must still be determined which thrust hemisphere contains the initial $q$ and which the $\bar{q}$.

In the case of the heaviest flavors accessible at the $Z^{0}, q=b, c$, the event flavor can be determined by the presence of a secondary vertex of high or low invariant mass, respectively. We selected samples of $96 \% b \bar{b}$ purity and $69 \% c \bar{c}$ purity, respectively, and measured these purities in the data. We have used four methods so far to determine the primary $b$ or $c$ direction, summarized in table 1 .

A standard method is to use identified $e^{ \pm}$and $\mu^{ \pm}$, which were assigned to $B$ decays, $D$ decays, or cascade $B \rightarrow D \rightarrow l$ decays based on the vertex mass and the lepton $p$ and $p_{t}$ with respect to the vertex flight direction. An $e^{-}$or $\mu^{-}$tags a $\bar{B}$ or $\bar{D}$ meson, and a likelihood fit was used to extract $A_{b}$ and $A_{c}$ simultaneously. This method is competitive due to the CRID-assisted $e^{ \pm}$and $\mu^{ \pm}$identification (sec. 2) and the high $P_{\text {corr }}$. However the efficiency is low and the sources of leptons overlap substantially, making it difficult to measure $P_{\text {corr }}$ in the data.

We have measured $A_{c}$ using $D$ and $D^{*}$ mesons reconstructed in several decay modes. The CRID is essential for the $D^{0} \rightarrow K^{-} \pi^{+}$(with no $D^{*}$ requirements) and $D_{s} \rightarrow$ $K^{+} K^{-} \pi^{+}$modes (see fig. 3). This method has essentially unit $P_{\text {corr }}$, but suffers from relatively low statistics.

A standard method for $A_{b}$ is the momentum-weighted event charge $Q$ [11], which gives 


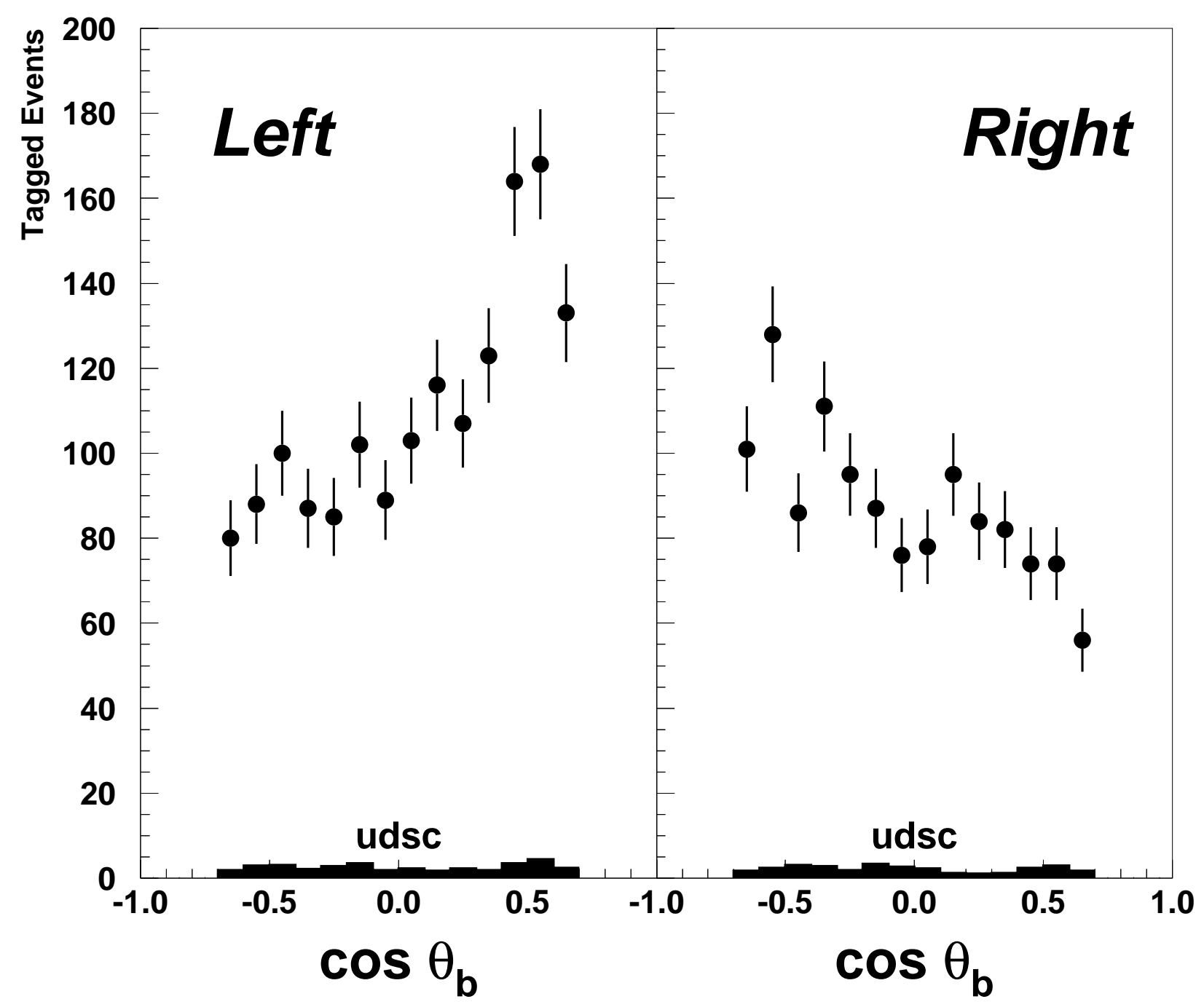

Figure 9: Distributions of the $b$-quark polar angle for events produced with negatively (left) and positively (right) polarized electron beams.

a sign to every tagged $b \bar{b}$ event, but $P_{\text {corr }}$ is low, averaging $\sim 60 \%$, and depends on $|Q|$. $P_{\text {corr }}$ can be measured in the data under some model-dependent assumptions, but the method will reach a systematic limit of a few percent.

We have recently demonstrated the use of identified $K^{ \pm}$to measure both $A_{b}$ [12] and $A_{c}$ 13. A $K^{ \pm}$was identified in $\sim 40 \%$ of the tagged $B$ hadron vertices with $P_{\text {corr }}=73 \%$, a smaller value than in sec. 4.1 since $B^{0}-\bar{B}^{0}$ mixing is now a dilution. This method provides a nice balance between efficiency and purity, yields the $\cos \theta_{b}$ distributions in fig. 9, and $P_{\text {corr }}$ can be measured unambigusouly in the data. The precision of the $P_{\text {corr }}$ measurement depends on the square of the efficiency and is also quite sensitive to background, so that CRID type $K^{ \pm}$identification is essential. Currently this measurement is statistics limited, as we have analyzed less than one-sixth of our data sample; we expect to measure $P_{\text {corr }}$ 
to $\pm 2 \%$, a valuable result in itself for future $B$ physics experiments, and to achieve one of the best precisions on $A_{b}$. Furthermore, this is a unique method complementary to those used so far, which give a world average value of $A_{b}$ that differs from the SM prediction by $3 \sigma$.

The situation is even better for charm. A $K^{ \pm}$was found in $\sim 40 \%$ of low mass vertices with zero net charge, and gave $P_{\text {corr }}>90 \%$. In charged vertices the vertex charge was combined with that of any identified $K^{ \pm}$to give an average $P_{\text {corr }}=91 \%$ for all charm vertices, and the $\cos \theta_{c}$ distributions shown in fig. 10. This $P_{\text {corr }}$ can be estimated reliably from known charmed hadron branching ratios, and with one-half of our data analyzed, we have the world's best measurement of $A_{c}$. A measurement of $P_{\text {corr }}$ from the data is feasible and will be required to reach $1 \%$ precision.

\section{Light Flavor Asymmetries}

In contrast to the situation with leptons or heavy flavors, there are published measurements of $Z^{0}$ couplings to light-flavor quarks $(u, d$ and $s$ ) only from DELPHI [14] and OPAL [15], with rather poor precision. The challenge is to separate these flavors not only from the heavy flavors but also from each other. Leading particles at high momentum can be used to determine the event flavor, and, if they carry the appropriate quantum number, the direction of the quark. However, the lack of experimental measurements of leading particle effects for these flavors leads to the choice of relying on a hadronization model to predict sample purities and $P_{\text {corr }}$, or trying to measure these in the data.

We have taken the approach of applying hard flavor selection cuts to reduce backgrounds, increase the $P_{\text {corr }}$ and therefore reduce the associated systematic errors. A hadronization model was used to predict these, but key quantities were measured in the data, the predictions adjusted accordingly, and the data statistics used to estimate the systematic errors.

To measure $A_{s}$ [16], we used the light flavor sample and tagged $s(\bar{s})$ hemispheres using identified $K^{-}\left(K^{+}\right)$with $p>9 \mathrm{GeV} / \mathrm{c}$ and reconstructed $\Lambda^{0} \rightarrow \mathrm{p} \pi^{-}\left(\bar{\Lambda}^{0} \rightarrow \overline{\mathrm{p}} \pi^{+}\right)$ with $p>5 \mathrm{GeV} / \mathrm{c}$ and the (anti)proton identified in the CRID. Requiring either an $s$-tag in one hemisphere and an $\bar{s}$-tag in the other, or an $s$ - or $\bar{s}$-tag in one hemisphere and a reconstructed $K_{s}^{0} \rightarrow \pi^{+} \pi^{-}$with $p>5 \mathrm{GeV} / \mathrm{c}$ in the other, yielded an event sample with $69 \% s \bar{s}$ purity and an average $P_{\text {corr }}=82 \%$. The distributions of $\cos \theta_{s}$ are shown in fig. 11 .

The heavy-flavor background is substantial but understood, and the associated systematic errors are small. $P_{\text {corr }}$ for $s \bar{s}$ events was measured in the data by counting hemispheres in which we identified three $K^{ \pm}$or $K_{s}^{0}$, since hemispheres with three true kaons are the dominant source of wrong signs. Similarly, the level and asymmetry of the $u \bar{u} / d \bar{d}$ background were measured by counting hemispheres with two identified kaons, and events with an $s$-tag (or $\bar{s}$-tag) in both hemispheres. The simulation was used to relate these counts to the relevant quantities, and was checked against our measured $K K$ correlations (sec. 3). 

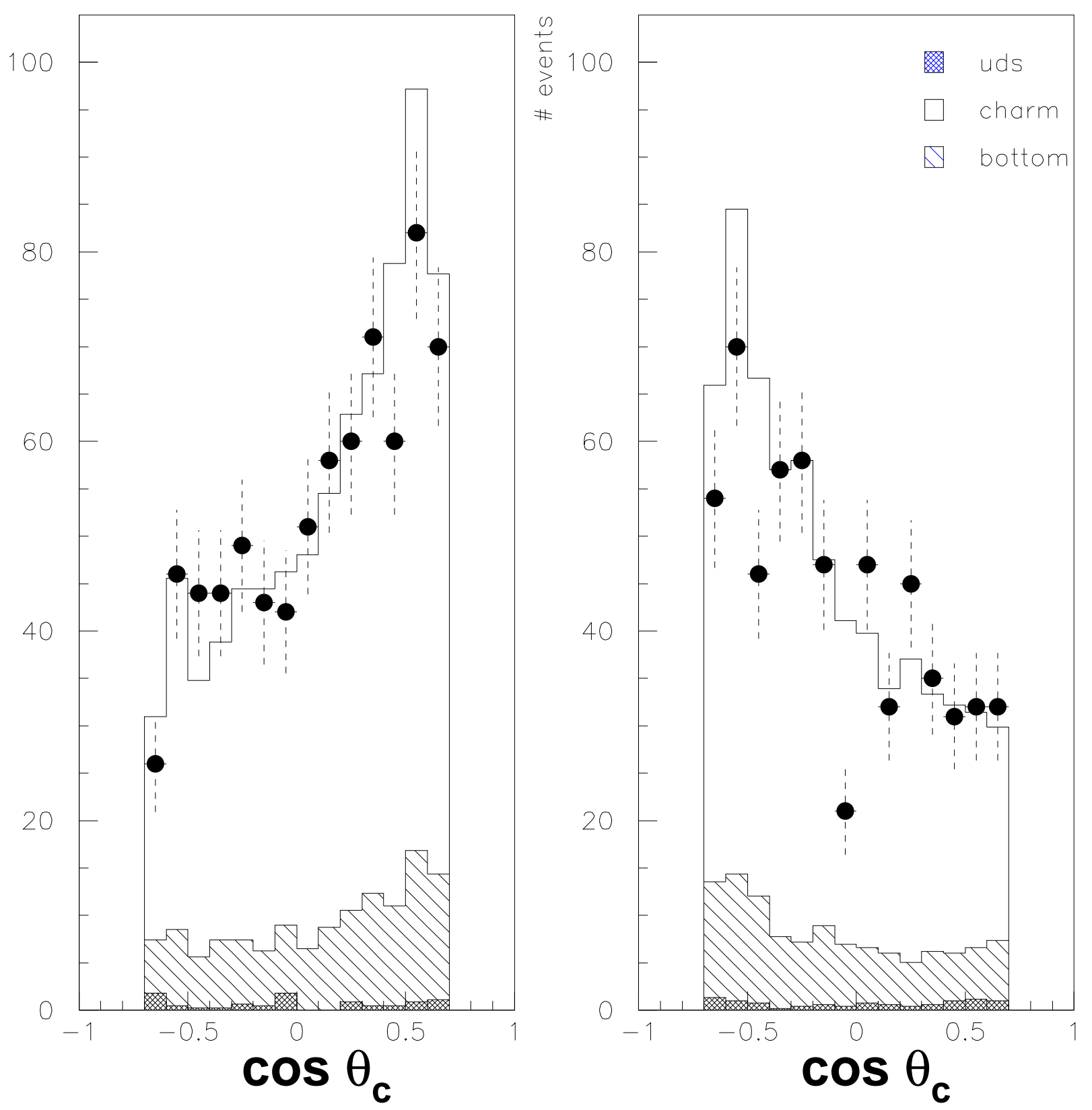

Figure 10: Distributions of the $c$-quark polar angle for left- and right-polarized beams. 

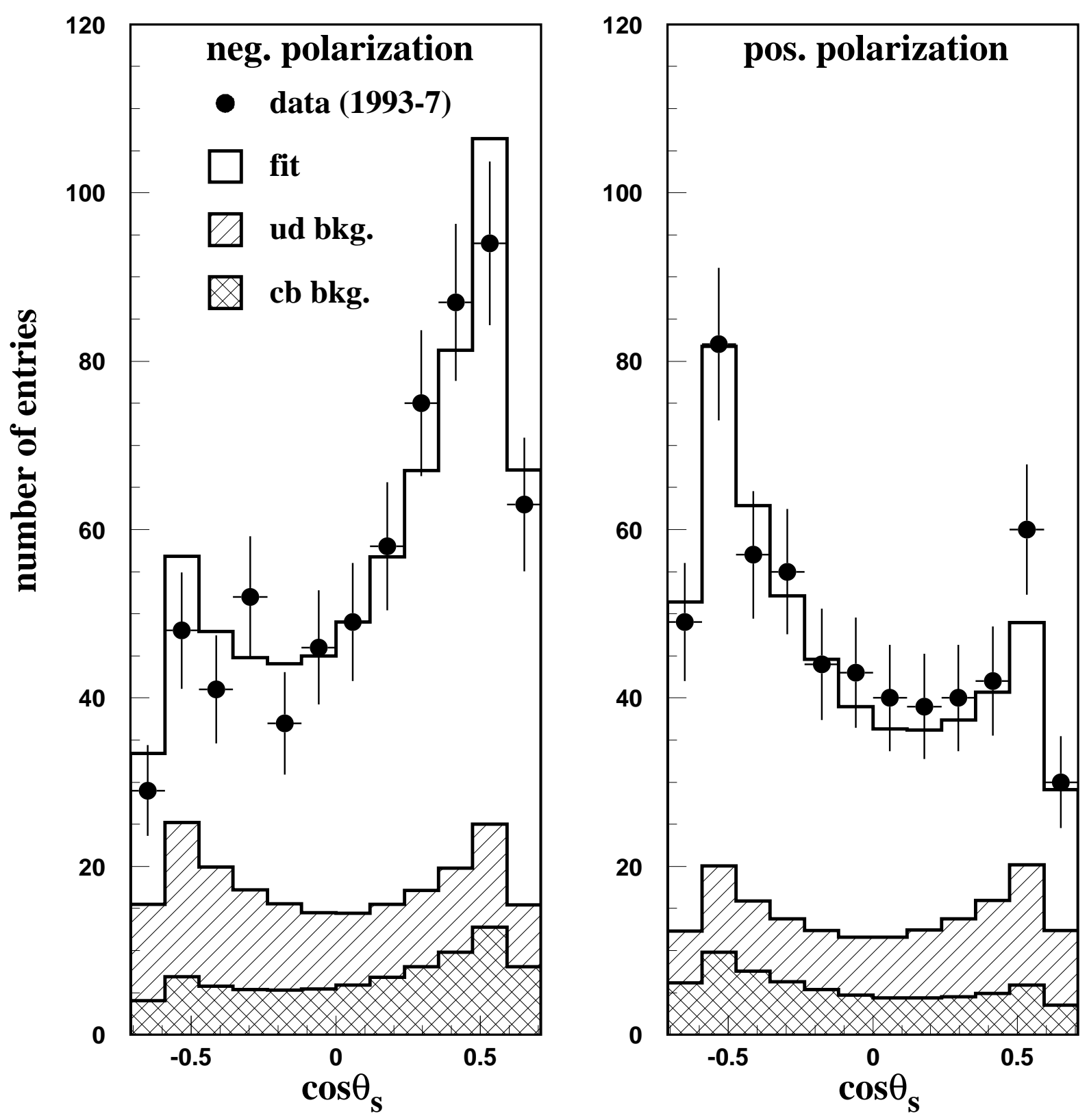

Figure 11: Distributions of the $s$-quark polar angle for left- and right-polarized beams. 
The CRID was essential in this analysis, yielding both a clean $s \bar{s}$ sample and enough 2- and 3-kaon hemispheres to measure the $P_{\text {corr }}$ and background. A fit gave $A_{s}=0.82 \pm$ $0.10 \pm 0.07$ (Preliminary), which is consistent with both the SM and the world average $A_{b}$, and is already a useful test of down-type universality. It is consistent with previous measurements and with a new measurement from DELPHI [17]. We expect a total relative uncertainty of $\sim 9 \%$ when our full data sample is analyzed, and with another data run or further input from the LEP experiments we might hope for a world average with a 5\% uncertainty

An open question is the extent to which we can measure the other two light flavor asymmetries, $A_{u}$ and $A_{d}$. The two flavors are expected to have similar production of leading pions and protons, but perhaps to differ in leading strange particle production. Here it is essential to measure the purities and $P_{\text {corr }}$ in the data, as has been done by OPAL [15]. We are pursuing this topic and expect $\sim 20 \%$ measurements based on the statitstics of our long-range correlation data (fig. (7).

\section{Conclusions}

We have presented a number of recent results from the SLD collaboration that use the CRID for charged particle identification. The performance of both the liquid and gaseous radiator systems has reached essentially the design parameters in terms of Cherenkov photon yield, angular resolution and hadron identification. CRID and calorimeter information have been combined to enhance the identification of electrons and muons. A wide variety of physics has been made possible or improved by the use of the CRID, in such diverse areas as hadronic jet structure, $B$-hadron decays and precision electroweak physics.

We have made precise studies of the production of $\pi^{ \pm}, K^{ \pm}, \mathrm{p} / \overline{\mathrm{p}}, K^{* 0}$ and $\phi$ in inclusive hadronic events, complementing measurements made with other particle identification techniques. We have also made new studies of events of different flavors and of leading particles. We have made the first precise studies of short-range, long-range and ordered correlations between pairs of identified $\pi^{ \pm}, K^{ \pm}$and $\mathrm{p} / \overline{\mathrm{p}}$ in light-flavor events. This set of results greatly enhances our understanding of jet formation.

The complete momentum coverage of $K^{ \pm}$from $B$ decays, combined with precision vertexing, has allowed a number of studies of $B$ hadron decays that are difficult at existing $\Upsilon(4 \mathrm{~S})$ detectors. We have studied the endpoint of the $K^{ \pm}$spectrum, finding no evidence for $b \rightarrow s g$ transitions. We have compared $K^{ \pm}$production from the up- and downstream vertices in inclusive $B$ decays, and extracted a new measurement of the fraction of doubly charmed $B$ decays. Such results demonstrate the power of full momentum coverage, and bode well for future dedicated $B$ physics experiments that include a RICH.

Flavor tagging of hadronic jets is an important recent advance in elementary particle physics. We have pioneered the use of $K^{ \pm}$to distinguish $B$ from $\bar{B}$ hadrons and $b$ from $\bar{b}$ jets inclusively, envisioned as an efficient and powerful tool at future dedicated $B$ physics 
experiments. Our measurements of $\Delta m_{d}$ and $A_{b}$ are competitive, with uncertainties dominated by the unknown analyzing power of this technique. When all our data are analyzed we expect to measure this analyzing power to $\pm 2 \%$ and to obtain measurements of $\Delta m_{d}$, $\Delta m_{s}$ and $A_{b}$ that are among the world's best and complement existing techniques. This inclusive method is also effective for charm; we already have the world's best measurement of $A_{c}$, and this technique should find applications at any future experiment that produces charm and includes a RICH.

We have established the tagging of $s$ jets using leading, high-momentum identified $K^{-}$and $\Lambda^{0}$ and produced the world's best measurement of $A_{s}$. Our studies of long-range correlations indicate that tagging of $u$ and $d$ jets using leading particles is possible, and we hope to make a complete set of measurements of the electroweak couplings of the $Z^{0}$

to the quarks. The potential applications for light-flavor tagging are numerous, including decays of $W^{ \pm}$bosons, top quarks, Higgs bosons, and new particles, as well as in the study of jet production in deep inelastic scattering and hadron-hadron collisions.

\section{References}

[1] SLD Design Report, SLAC-Report 273 (1984).

[2] M. Battaglia, to appear in Proceedings of the $3^{\text {rd }}$ International Workshop on Ring Imaging Cherenkov Detectors, Ein-Gedi, Israel, November 1998.

[3] SLD Collab., K. Abe et al., SLAC-PUB-7896, contributed to the XXIX ${ }^{\text {th }}$ ICHEP, Vancouver, Canada, July 1998, ICHEP-183; M. Daoudi et al., SLAC-PUB-7941, to appear in the proceedings of the XXIX ${ }^{\text {th }}$ ICHEP, Vancouver, Canada, July 1998.

[4] K. Abe et al., Nucl. Inst. Meth. A343 (1994) 74; J. Va'vra, to appear in Proceedings of the $3^{\text {rd }}$ International Workshop on Ring Imaging Cherenkov Detectors, Ein-Gedi, Israel, November 1998.

[5] K. Abe et al., Nucl. Inst. Meth. A371 (1996) 195.

[6] SLD Collab. K. Abe et al., SLAC-PUB-7766, Phys. Rev. D59 (1999) 052001.

[7] A. Böhrer, Phys. Rep. 291 (1997) 107.

[8] SLD Collab., K. Abe et al., Phys. Rev. Lett. 78 (1997) 3442.

[9] SLD Collab., K. Abe et al., SLAC-PUB-7824, contributed to the XXIX ${ }^{\text {th }}$ ICHEP, Vancouver, Canada, July 1998, ICHEP-259.

[10] D. Jackson, Nucl. Inst. Meth. A388 (1997) 247.

[11] SLD Collab., K. Abe et al., Phys. Rev. Lett. 81 (1998) 942. 
[12] SLD Collab., K. Abe et al., SLAC-PUB-7766, submitted to Physical Review Letters.

[13] SLD Collab., K. Abe et al., SLAC-PUB-7879, contributed to the XXIX ${ }^{\text {th }}$ ICHEP, Vancouver, Canada, July 1998, ICHEP-175.

[14] DELPHI Collab., P. Abreu et al., Z. Phys. C67 (1995) 1.

[15] OPAL Collab., K. Ackerstaff et al., Z. Phys. C76 (1997) 387.

[16] SLD Collab., K. Abe et al., SLAC-PUB-7825, contributed to the XXIX ${ }^{\text {th }}$ ICHEP, Vancouver, Canada, July 1998, ICHEP-254.

[17] M. Gunther, to appear in Proceedings of the $3^{\text {rd }}$ International Workshop on Ring Imaging Cherenkov Detectors, Ein-Gedi, Israel, November 1998.

\section{${ }^{* *}$ List of Authors}

K. Abe ${ }^{(2)}$ K. Abe, ${ }^{(19)}$ T. Abe, ${ }^{(27)}$ I.Adam,${ }^{(27)}$ T. Akagi, ${ }^{(27)}$ N. J. Allen, ${ }^{(4)}$ A. Arodzero, ${ }^{(20)}$ W.W. Ash ${ }^{(27)}$ D. Aston, ${ }^{(27)}$ K.G. Baird,${ }^{(15)}$ C. Baltay ${ }^{(37)}$ H.R. Band, ${ }^{(36)}$ M.B. Barakat, ${ }^{(14)}$ O. Bardon, ${ }^{(17)}$ T.L. Barklow, ${ }^{(27)}$ J.M. Bauer, ${ }^{(16)}$ G. Bellodi, ${ }^{(21)}$ R. Ben-David, ${ }^{(37)}$ A.C. Benvenuti, ${ }^{(3)}$ G.M. Bilei ${ }^{(23)}$ D. Bisello, ${ }^{(22)}$ G. Blaylock, ${ }^{(15)}$ J.R. Bogart, ${ }^{(27)}$ B. Bolen, ${ }^{(16)}$ G.R. Bower, ${ }^{(27)}$ J. E. Brau ${ }^{(20)}$ M. Breidenbach, ${ }^{(27)}$ W.M. Bugg, ${ }^{(30)}$ D. Burke, ${ }^{(27)}$ T.H. Burnett ${ }^{(35)}$ P.N. Burrows, ${ }^{(21)}$ A. Calcaterra, ${ }^{(11)}$ D.O. Caldwell, ${ }^{(32)}$ D. Calloway, ${ }^{(27)}$ B. Camanzi, ${ }^{(10)}$ M. Carpinelli, ${ }^{(24)}$ R. Cassell, ${ }^{(27)}$ R. Castaldi, ${ }^{(24)}$ A. Castro, ${ }^{(22)}$ M. Cavalli-Sforza ${ }^{\left({ }^{(3)}\right)}$ A. Chou, ${ }^{(27)}$ E. Church, ${ }^{(35)}$ H.O. Cohn, ${ }^{(30)}$ J.A. Coller, ${ }^{(5)}$ M.R. Convery, ${ }^{(27)}$ V. Cook, ${ }^{(35)}$ R. Cotton, ${ }^{(4)}$ R.F. Cowan, ${ }^{(17)}$ D.G. Coyne, ${ }^{(33)}$ G. Crawford, ${ }^{(27)}$ C.J.S. Damerell, ${ }^{(25)}$

M. N. Danielson, ${ }^{(7)}$ M. Daoudi, ${ }^{(27)}$ N. de Groot, ${ }^{(27)}$ R. Dell'Orso, ${ }^{(23)}$ P.J. Dervan, ${ }^{(4)}$ R. de Sangro, ${ }^{(11)}$ M. Dima, ${ }^{(9)}$ A. D'Oliveira, ${ }^{(6)}$ D.N. Dong, ${ }^{(17)}$ P.Y.C. Du, ${ }^{(30)}$ R. Dubois, ${ }^{(27)}$ B.I. Eisenstein, ${ }^{(12)}$ V. Eschenburg, ${ }^{(16)}$ E. Etzion, ${ }^{(36)}$ S. Fahey, ${ }^{(7)}$ D. Falciai, ${ }^{(11)}$ C. Fan, ${ }^{(7)}$ J.P. Fernandez, ${ }^{(33)}$ M.J. Fero, ${ }^{(17)}$ K.Flood, ${ }^{(15)}$ R. Frey ${ }^{(20)}$ T. Gillman, ${ }^{(25)}$ G. Gladding, ${ }^{(12)}$ S. Gonzalez, ${ }^{(17)}$ E.L. Hart ${ }^{(30)}$ J.L. Harton, ${ }^{(9)}$ A. Hasan, ${ }^{(4)}$ K. Hasuko, ${ }^{(31)}$ S. J. Hedges, ${ }^{(5)}$ S.S. Hertzbach, ${ }^{(15)}$ M.D. Hildreth, ${ }^{(27)}$ J. Huber, ${ }^{(20)}$ M.E. Huffer, ${ }^{(27)}$ E.W. Hughes, ${ }^{(27)}$ X.Huynh,${ }^{(27)}$ H. Hwang, ${ }^{(20)}$ M. Iwasaki, ${ }^{(20)}$ D. J. Jackson, ${ }^{(25)}$ P. Jacques, ${ }^{(26)}$ J.A. Jaros, ${ }^{(27)}$ Z.Y. Jiang, ${ }^{(27)}$ A.S. Johnson, ${ }^{(27)}$ J.R. Johnson, ${ }^{(36)}$ R.A. Johnson, ${ }^{(6)}$ T. Junk, ${ }^{(27)}$ R. Kajikawa, ${ }^{(19)}$ M. Kalelkar, ${ }^{(26)}$ Y. Kamyshkov, ${ }^{(30)}$ H.J. Kang, ${ }^{(26)}$ I. Karliner ${ }^{(12)}$ H. Kawahara, ${ }^{(27)}$ Y. D. Kim, ${ }^{(28)}$ R. King, ${ }^{(27)}$ M.E. King, ${ }^{(27)}$ R.R. Kofler, ${ }^{(15)}$ N.M. Krishna,${ }^{(7)}$ R.S. Kroeger, ${ }^{(16)}$ M. Langston, ${ }^{(20)}$ A. Lath, ${ }^{(17)}$ D.W.G. Leith, ${ }^{(27)}$ V. Lia, ${ }^{(17)}$ C.-J. S. Lin, ${ }^{(27)}$ X. Liu, ${ }^{(33)}$ M.X. Liu ${ }^{(37)}$ M. Loreti, ${ }^{(22)}$ A. Lu ${ }^{(32)}$ H.L. Lynch,,${ }^{(27)}$ J. Ma ${ }^{(35)}$ G. Mancinelli, ${ }^{(26)}$ S. Manly, ${ }^{(37)}$ G. Mantovani, ${ }^{(23)}$ T.W. Markiewicz, ${ }^{(27)}$ T. Maruyama, ${ }^{(27)}$ H. Masuda, ${ }^{(27)}$ E. Mazzucato, ${ }^{(10)}$ A.K. McKemey, ${ }^{(4)}$ B.T. Meadows, ${ }^{(6)}$ G. Menegatti, ${ }^{(10)}$ R. Messner, ${ }^{(27)}$ P.M. Mockett, ${ }^{(35)}$ K.C. Moffeit, ${ }^{(27)}$ T.B. Moore, ${ }^{(37)}$ M.Morii, ${ }^{(27)}$ D. Muller, ${ }^{(27)}$ V.Murzin, ${ }^{(18)}$ T. Nagamine, ${ }^{(31)}$ S. Narita, ${ }^{(31)}$ U. Nauenberg, ${ }^{(7)}$ H. Neal, ${ }^{(27)}$ M. Nussbaum ${ }^{(6)}$ N.Oishi, ${ }^{(19)}$ D. Onoprienko, ${ }^{(30)}$ L.S. Osborne,${ }^{(17)}$ 
R.S. Panvini, ${ }^{(34)}$ H. Park, ${ }^{(20)}$ C. H. Park, ${ }^{(29)}$ T.J. Pavel, ${ }^{(27)}$ I. Peruzzi, ${ }^{(11)}$ M. Piccolo, ${ }^{(11)}$ L. Piemontese, ${ }^{(10)}$ E. Pieroni, ${ }^{(24)}$ K.T. Pitts, ${ }^{(20)}$ R.J. Plano, ${ }^{(26)}$ R. Prepost, ${ }^{(36)}$ C.Y. Prescott, ${ }^{(27)}$ G.D. Punkar, ${ }^{(27)}$ J. Quigley, ${ }^{(17)}$ B.N. Ratcliff, ${ }^{(27)}$ T.W. Reeves, ${ }^{(34)}$ J. Reidy, ${ }^{(16)}$ P.L. Reinertsen, ${ }^{(33)}$ P.E. Rensing, ${ }^{(27)}$ L.S. Rochester, ${ }^{(27)}$ P.C. Rowson, ${ }^{(8)}$ J.J. Russell, ${ }^{(27)}$ O.H. Saxton, ${ }^{(27)}$ T. Schalk, ${ }^{(33)}$ R.H. Schindler, ${ }^{(27)}$ B.A. Schumm, ${ }^{(33)}$ J. Schwiening, ${ }^{(27)}$ S. Sen, ${ }^{(37)}$ V.V. Serbo, ${ }^{(36)}$ M.H. Shaevitz, ${ }^{(8)}$ J.T. Shank, ${ }^{(5)}$ G. Shapiro, ${ }^{(13)}$ D.J. Sherden, ${ }^{(27)}$ K. D. Shmakov, ${ }^{(30)}$ C. Simopoulos, ${ }^{(27)}$ N.B. Sinev, ${ }^{(20)}$ S.R. Smith, ${ }^{(27)}$ M. B. Smy, ${ }^{(9)}$ J.A. Snyder, ${ }^{(37)}$ H. Staengle, ${ }^{(9)}$ A. Stahl, ${ }^{(27)}$ P. Stamer, ${ }^{(26)}$ R. Steiner, ${ }^{(1)}$ H. Steiner, ${ }^{(13)}$ M.G. Strauss, ${ }^{(15)}$ D. Su, ${ }^{(27)}$ F. Suekane, ${ }^{(31)}$ A. Sugiyama, ${ }^{(19)}$ S. Suzuki, ${ }^{(19)}$ M. Swartz, ${ }^{(27)}$ A. Szumilo, ${ }^{(35)}$ T. Takahashi, ${ }^{(27)}$ F.E. Taylor, ${ }^{(17)}$ J. Thom, ${ }^{(27)}$ E. Torrence, ${ }^{(17)}$ N. K. Toumbas, ${ }^{(27)}$ A.I. Trandafir, ${ }^{(15)}$ J.D. Turk, ${ }^{(37)}$ T. Usher, ${ }^{(27)}$ C. Vannini, ${ }^{(24)}$ J. Va'vra, ${ }^{(27)}$ E. Vella, ${ }^{(27)}$ J.P. Venuti, ${ }^{(34)}$ R. Verdier, ${ }^{(17)}$ P.G. Verdini, ${ }^{(24)}$ S.R. Wagner, ${ }^{(27)}$ D. L. Wagner, ${ }^{(7)}$ A.P. Waite, ${ }^{(27)}$ Walston, S. ${ }^{(20)}$ J.Wang, ${ }^{(27)}$ C. Ward, ${ }^{(4)}$ S.J. Watts, ${ }^{(4)}$ A.W. Weidemann, ${ }^{(30)}$ E. R. Weiss, ${ }^{(35)}$ J.S. Whitaker, ${ }^{(5)}$ S.L. White, ${ }^{(30)}$ F.J. Wickens, ${ }^{(25)}$ B. Williams, ${ }^{(7)}$ D.C. Williams, ${ }^{(17)}$ S.H. Williams, ${ }^{(27)}$ S. Willocq, ${ }^{(27)}$ R.J. Wilson, ${ }^{(9)}$ W.J. Wisniewski, ${ }^{(27)}$ J. L. Wittlin, ${ }^{(15)}$ M. Woods, ${ }^{(27)}$ G.B. Word, ${ }^{(34)}$ T.R. Wright, ${ }^{(36)}$ J. Wyss, ${ }^{(22)}$ R.K. Yamamoto, ${ }^{(17)}$ J.M. Yamartino, ${ }^{(17)}$ X. Yang, ${ }^{(20)}$ J. Yashima, ${ }^{(31)}$ S.J. Yellin, ${ }^{(32)}$ C.C. Young, ${ }^{(27)}$ H. Yuta, ${ }^{(2)}$ G. Zapalac, ${ }^{(36)}$ R.W. Zdarko, ${ }^{(27)}$ J. Zhou. ${ }^{(20)}$

(1) Adelphi University, South Avenue, Garden City, NY 11530

(2) Aomori University, 2-3-1 Kohata, Aomori City, 030 Japan

(3) INFN Sezione di Bologna, Via Irnerio 46, I-40126 Bologna, Italy

(4) Brunel University, Uxbridge, Middlesex, UB8 3PH United Kingdom

${ }^{(5)}$ Boston University, 590 Commonwealth Ave., Boston, MA 02215

(6) University of Cincinnati, Cincinnati, OH 45221

(7) University of Colorado, Campus Box 390, Boulder, CO 80309

(8) Columbia University, Nevis Laboratories, P.O. Box 137, Irvington, NY 10533

(9) Colorado State University, Ft. Collins, CO 80523

(10) INFN Sezione di Ferrara, Via Paradiso 12, I-44100 Ferrara, Italy

(11) Lab. Nazionali di Frascati, Casella Postale 13, I-00044 Frascati, Italy

(12) University of Illinois, 1110 West Green St., Urbana, IL 61801

(13) Lawrence Berkeley Laboratory, Dept. of Physics, 50B-5211 University of California, Berkeley, CA 94720

${ }^{(14)}$ Louisiana Technical University, Ruston, LA 71272

(15) University of Massachusetts, Amherst, MA 01003

(16) University of Mississippi, University, MS 3867r

(17) Massachusetts Institute of Technology, $77^{7}$ Massachussetts Avenue, Cambridge, MA 02139

(18) Moscow State University, Institute of Nuclear Physics, 119899 Moscow, Russia

${ }^{(19)}$ Nagoya University, Nagoya 464, Japan

(20) University of Oregon, Department of Physics, Eugene, OR 97403

(21) Oxford University, Oxford, OX1 3RH, United Kingdom

(22) Universita di Padova, Via F. Marzolo 8, I-35100 Padova, Italy

(23) Universita di Perugia, Sezione INFN, Via A. Pascoli, I-06100 Perugia, Italy 
(24) INFN, Sezione di Pisa, Via Livornese 582/AS, Piero a Grado, I-56010 Pisa, Italy

${ }^{(25)}$ Rutherford Appleton Laboratory, Chilton, Didcot, Oxon, OX11 0QX United Kingdom

${ }^{(26)}$ Rutgers University, Serin Physics Labs., Piscataway, NJ 08855

(27) Stanford Linear Accelerator Center, 2575 Sand Hill Road, Menlo Park, CA 94025

(28) Sogang University, Ricci Hall, Seoul, Korea

(29) Soongsil University, Seoul, Korea 156-743

(30) University of Tennessee, 401 A.H. Nielsen Physics Blg., Knoxville, TN 37996

(31) Tohoku University, Bubble Chamber Lab., Aramaki, Sendai 980, Japan

${ }^{(32)}$ U.C. Santa Barbara, 3019 Broida Hall, Santa Barbara, CA ,93106

${ }^{(33)}$ U.C. Santa Cruz, Santa Cruz,,CA ,95064

(34) Vanderbilt University, Stevenson Center, P.O.Box 180\%, Station B, Nashville, TN 37235

(35) University of Washington, Seattle, WA 98105

(36) University of Wisconsin, 1150 University Avenue, Madison, WI 53706

(37) Yale University, 5th Floor Gibbs Lab., P.O.Box 208121, New Haven, CT 06520 